\title{
Saurositus peyrierasi n.sp., Filaire parasite d'Uroplatus fimbriatus à Madagascar
} par J. PROD'HON

Centre O.R.S.T.O.M., Tananarive (Madagascar)

\begin{abstract}
Résumé
Une nouvelle espèce de Saurositus, $S$. peyrierasi n. sp., parasite d'Uroplate est décrite à Madagascar; elle présente davantage d'affinités avec l'espèce indienne qu'avec les espèces africaines.
\end{abstract}

\section{Summary}

A new species of Saurositus, S. peyrierasi n. sp., parasite of Uroplatus is described from Madagascar; it seems more closely related to indian sp. than to african species.

L'autopsie de deux Uroplatus fimbriatus (Schneider, 1797) provenant de NosyMangabe, petite île située au fond de la baie d'Antongil (Madagascar), a permis de récolter plusieurs mâles et femelles d'une nouvelle espèce de Filaire. Ces Nématodes étaient localisés sous la peau et dans la cavité générale.

\section{Matériel étudié.}

Six femelles dont la longueur varie de 38 à $23,8 \mathrm{~mm}(38 \mathrm{~mm}, 34,4 \mathrm{~mm}, 34,1 \mathrm{~mm}$, $30,1 \mathrm{~mm}, 28 \mathrm{~mm}$ et $23,8 \mathrm{~mm}$ ) et la largeur, mesurée à la partie moyenne du corps, de 210 à $190 \mu$. Deux mâles mesurant 21,9 et $19,4 \mathrm{~mm}$ de long sur $150 \mu$ de large à la partie moyenne du corps.

\section{Deseription.}

Corps fin sans ailes ni crêtes internes latérales, s'amincissant brusquement au niveau de la jonction œsophage-intestin. Bouche simple, sans lèvres, approximativement triangulaire. Cavité buccale triangulaire. Tête portant deux amphides, quatre papilles bien visibles et près de ia bouche quatre papilles soudées deux à deux (fig. II A, B). 
(Esophage formé d'une portion musculaire et d'une portion glandulaire plus large et beaucoup plus longue (fig. III E). Lumière de l'œsophage comprimée sur toute la longueur (fig. II C). Les orientations différentes de la lumière œsophagienne à chaque niveau de section montrent que l'œsophage tourne autour d'un axe longitudinal.
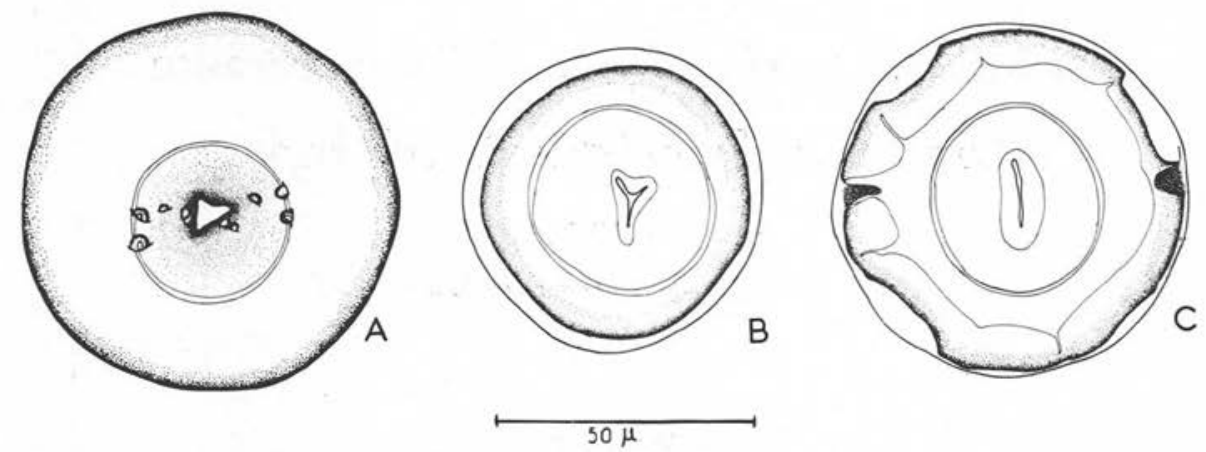

Fio. II. - A, vue apicale de la tête, femelle. B, coupe transversale au niveau de la capsule buccale. femelle. C, coupe transversale au niveau de l'œsophage, femelle. A, B, C, échelle $100_{\mu}$

- Femelle (fig. I).

Femel.e holotype mesurant $34,4 \mathrm{~mm}$ de long sur $200 \mu$ de large à la partie moyenne du corps. Cavité buccale très réduite. Anneau nerveux et pore excréteur respectivement à 140 et $580 \mu$ de l'extrémité antérieure. Déirides non vues. Esophage musculaire long de $180 \mu$. et œsophage glandulaire long de $605 \mu$. Vulve non saillante située approximativement au niveau de la jonction œsophage-intestin (sur le spécimen holotype vulve située à $775 \mu$ de l'extrémité antérieure). Ovéjecteur impair dirigé vers l'arrière. Division en deux utérus à $1.820 \mu$ de la vulve. Ovaires repliés dans la partie terminale du corps. Anus subterminal à $92 \mu$ de l'extrémité postérieure du corps. Sur certaines femelles, l'anus semble atrophié. Queue arrondie.

- Mâle (fig. III).

Spécimen allotype mesurant $21,9 \mathrm{~mm}$ de long sur $150 \mu$ de large à la partie moyenne du corps. Cavité buccale haute de $15 \mu$. Anneau nerveux et pore excréteur respectivement à 120 et $380 \mu$ de l'extrémité antérieure. Déirides non vues. Esophage musculaire long de $220 \mu$ et œsophage glandulaire long de $420 \mu$. Anus terminal. Queue longue de $5 \mu$. Extrémité postérieure enroulée sur 3-4 tours de spire. Région post-anale aplatie sur la face dorsale. Deux spicules de forme complexe, soudés à l'apex et évasés en corolle à leur partie proximale: longueur du spicule droit de $72 \mu$, sur une largeur de $12 \mu$ à la partie moyenne et $21 \mu$ à la partie terminale ; longueur du spicule gauche de $65 \mu$, sur une largeur de $9 \mu$ à la partie moyenne et $18 \mu$ à la partie terminale. Il existe, entre la partie subterminale des deux spicules, deux lames faiblement chitinisées, insérées sur le bord interne des spicules (fig. III C et D). La lame ventrale est de forme triangulaire, avec pointe en triangle dirigée vers l'anus et la lame dorsale est de forme trapézoïdale. Existence de cinq paires de papilles pré-anales (fig. III A et B). 


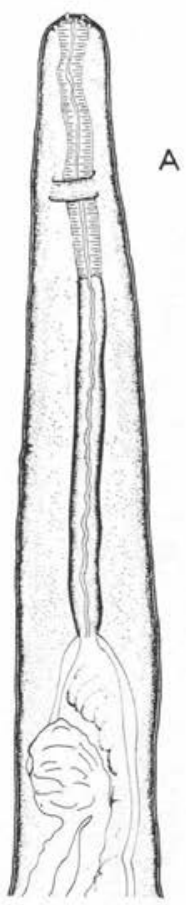

A
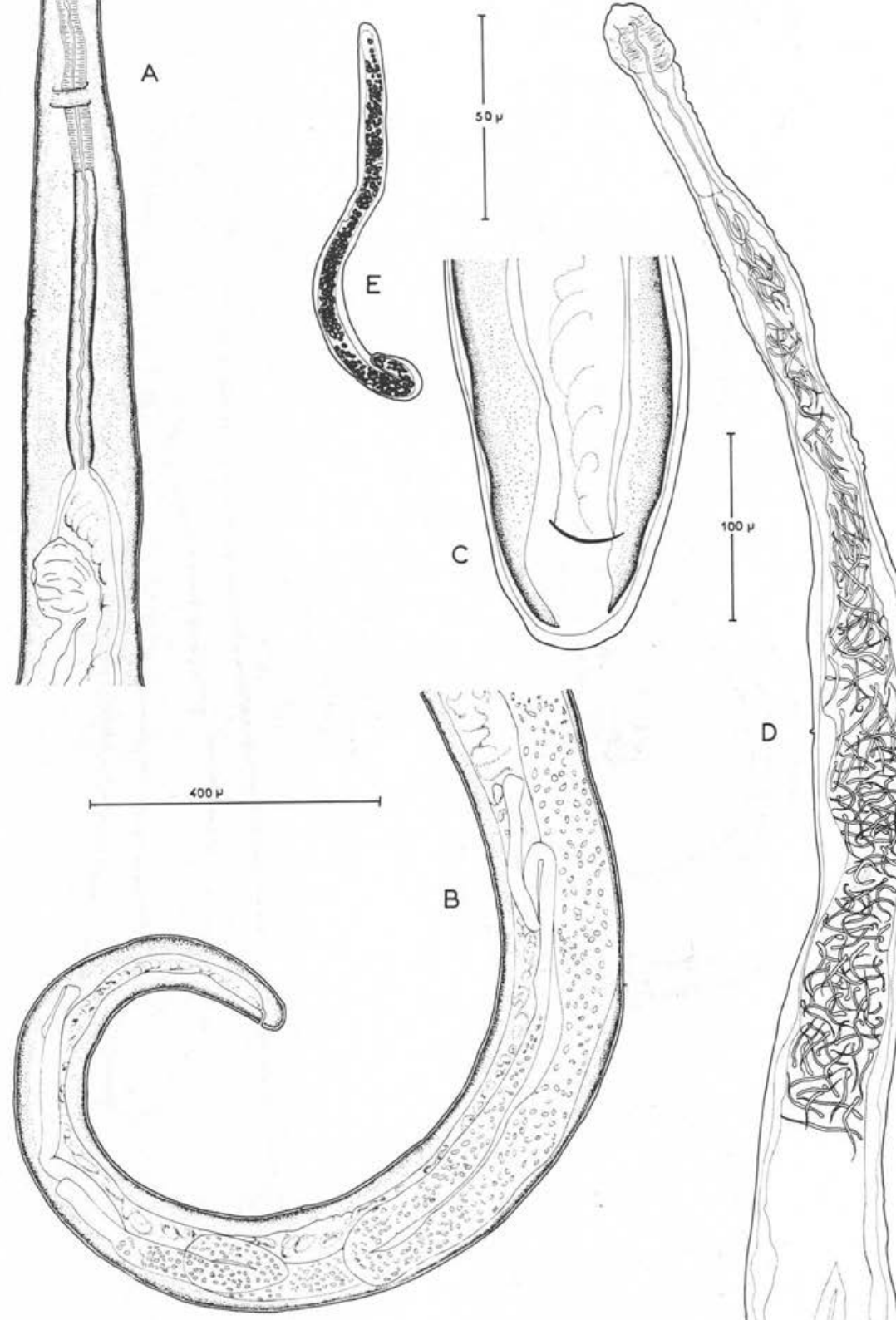

D

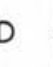

Fig. I. - A, extrémité antérieure de la femelle, vae latérale. B, queue de la femelle, vue latérale. $\mathrm{C}$, queue de la femelle, vue ventrale. $\mathrm{D}$, dissection de l'appareil génital femelle. E, microfilaire. A, B et D, échelle $400 u$; C, échelle $100_{u}$; E, échelle $100 u$. 


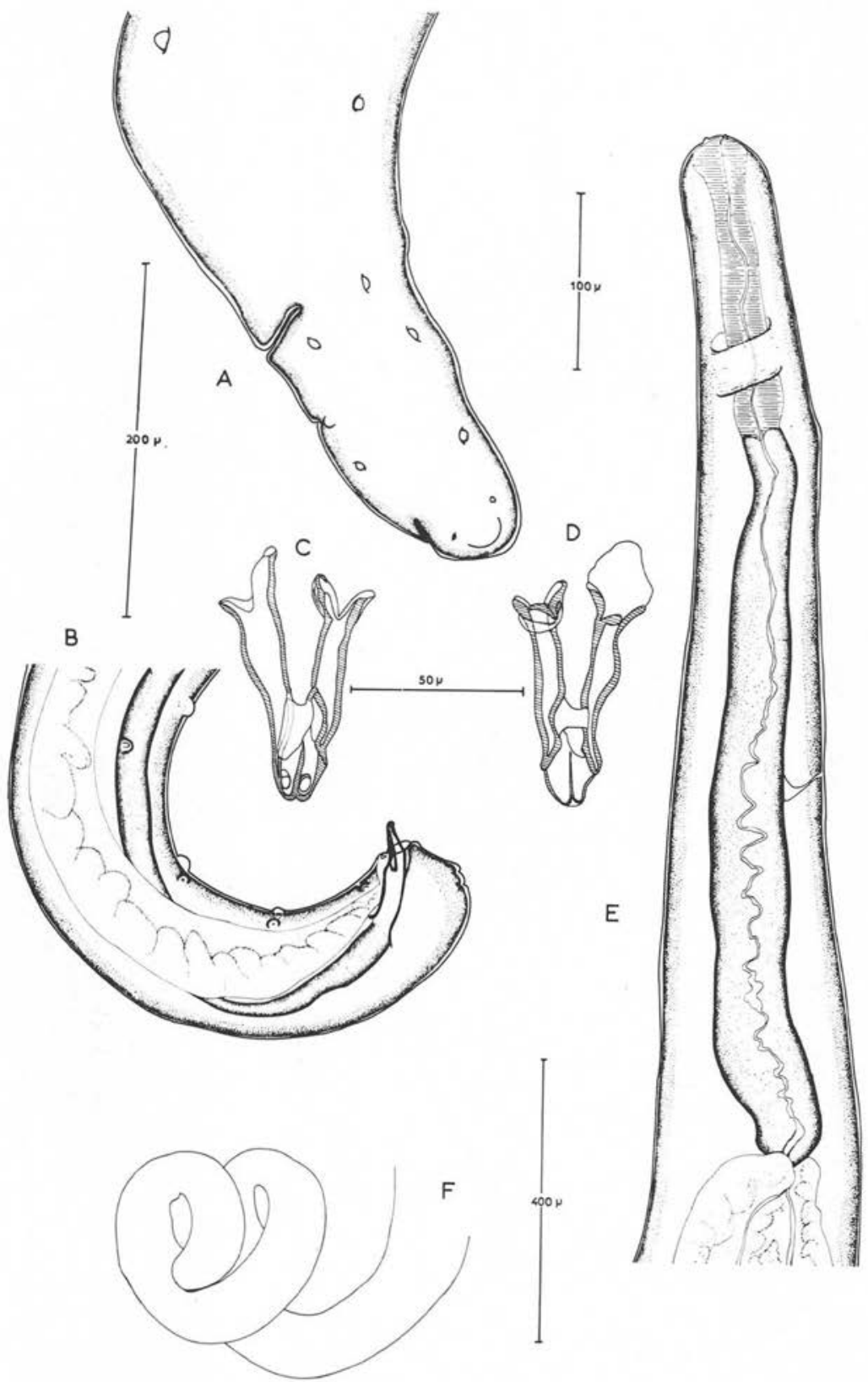

FIG. III. - A, queue du mâle, vue ventrale. B, queue du mâle, vue latérale. C, spicules, vue ventrale. D, Spicules, vue dorsale. E, extrémité antérieure du mâle, vue latérale. F, région caudale du mâle. $\mathrm{A}$ et $\mathrm{B}$, échelle $200 u ; \mathrm{C}$ et $\mathrm{D}$, échelle $50 u$; E, échelle $100 u ; \mathrm{F}$, échelle $400 u$. 
- Microfilaire (fig. I E).

Nous avons examiné les microfilaires après coloration au Giemsa sur goutte épaisse de sang. Longueur moyenne $130 \mu$ (entre 125 et $135 \mu$.). Largeur 5,5 $\mu$. Anneau nerveux et pore excréteur n'interrompant pas la colonne nucléaire et respectivement situés à 35 et $50 \mu$ (sur une microfilaire longue de $130 \mu$ ) de l'extrémité antérieure. Corps interne difficilement visible. Pore anal à $20 \mu$ de l'extrémité postérieure.

\section{Expérimentation.}

Nous avons tenté le cycle de cette Filaire avec Culex pipiens fatigans (trois essais: 81,7 et 106 moustiques gorgés) et Anopheles gambiae $B$ (trois essais : 2, 4 et 9 moustiques gorgés). Jusqu'ici les résultats ont été négatifs.

\section{Discussion.}

Nos spécimens présentent tous les caractères du genre Saurositus Macfie, 1924 [Eufilariinae Lopez-Neyra 1956, Onchocercidae (Leiper, 1911) Chabaud et Anderson, 1959]. A notre connaissance, c'est la première fois qu'est décrite une Filaire du genre Saurositus et chez un Geckonidae à Madagascar.

D'après Bain (1970), le genre comprend actuellement trois espèces: Saurositus indicus Deshmukh et Mehdi-Ali, 1965, chez Calotes versicolor aux Indes; Saurositus baal Sulahian et Schacher, 1969, chez Agama stellio au Liban; Saurositus agamae en Afrique, qui est un complexe de trois sous-espèces: Saurositus agamae agamae Macfie, 1924, chez Agama agama au Nigeria et en Côte-d'Or, dans la zone forestière, Saurositus agamae hamoni Bain, 1969, chez le même hôte en Haute-Volta, en zone de savane, et Saurositus agamae macfiei Fitzsimmons, 1957, chez Agama mossambica mossambica en Afrique orientale (Nyassaland) en altitude.

Nos spécimens, par leur tailie réduite et l'absence de crêtes cuticulaires latérales internes, se distinguent aisément du complexe $S$. agamae.

Les spécimens de Schacher sont beaucoup plus grands que les nôtres, mais avec des microfilaires plus petites.

Par leur petite taille, nos spécimens se rapprochent de $S$. indicus; mais ils s'en distinguent par des dimensions un peu plus élevées et par la forme différente des spicules.

Nos spécimens nous paraissent donc constituer une espèce nouvelle que nous dédions à M. Peyrieras; nous la nommons Saurositus peyrierasi n.sp.

\section{Conclusion.}

Comme dans beaucoup d'autres exemples, la forme malgache a donc plus d'affinités avec la forme indienne qu'avec les formes africaines.

Les caractères morphologiques sont trop pauvres et trop uniformes pour que l'on puisse établir des conclusions précises. Cependant, en prenant pour caractères la longueur du corps et celle des spicules, on obtient une sériation qui va de l'Inde à Madagascar, en Afrique orientale, en Afrique occidentale - forêt - et en Afrique 
occidentale - savane - la forme connue du Liban restant relativement proche de celle de l'Inde. On peut donc imaginer une extension de l'espèce, avec spéciations successives.

\section{Bibliographie}

BaIN (O.), 1969. - Développement larvaire de Saurositus agamae hamoni n. s. sp. Eufilariinae parasite d'Agame en Haute-Volta chez Anopheles stephensi. Ann. Parasit., 44, 581-594.

Deshmukh (P. G.) et Mehdi-Ali (S.), 1965. - On a new species of the genus Saurositus from an Indian lizard. J. Helmint., 39, 137-140.

Frtzsimmons (W. M.), 1958. - Saurositus macfiei n.sp., a filarioid parasite of the lizard Agama mossambica mossambica Peters. Ann. trop. Med. Parasit., 52, 257-260.

MACFIE (J.W. S.), 1914. - Notes on some blood parasites collected in Nigeria. Ann. trop. Med. Parasit., 8, 456-458.

-, 1924. - Saurositus agamae, n. g., n. sp., a filarioid parasite of the lizard Agama colonorum, Ann. trop. Med. Parasit., 18, 409-412.

Sulahian (A.) et Schacher (J. F.), 1969. - Saurositus baal n. sp., a filarial worm from the lizard Agama stellio in Lebanon, with notes on Saurositus macfiei Fitzsimmons, 1958. J. Parasit., 55, 105-107. 